\title{
On the Filtration Efficiency of Composite Media Composed of Multiple Layers of Electret Media
}

\section{Aerosol and Air Quality Research}

Peng Wang, Da-Ren Chen*

Particle Laboratory, Department of Mechanical and Nuclear Engineering, Virginia

Commonwealth University, Richmond, VA 23284, USA

\section{ABSTRACT}

This study aimed to explain the discrepancy reported in previous studies between the observed and the calculated filtration efficiencies of a composite formed of multiple electret medium layers. After measuring the composite's filtration efficiency using particles divided by size and electrical charge status, viz., those possessing no charge, a single charge, and a stationary charge according to the Boltzmann distribution, we traced the discrepancy to the latter attribute. Hence, to accurately predict the filtration efficiency of multi-layered electret media, we must account for the test particles' electrical charge distribution.

Keywords: Multi-layered electret media, Composite electret media, Filtration efficiency, Boltzmann stationary charge distribution

\section{INTRODUCTION}

Under the same pressure drop, electret fibrous media typically offer higher collection efficiencies compared to those of mechanical ones in the same microstructures because of the additional electrostatic particle capture mechanisms (i.e., Coulombic attraction and dielectrophoresis). Electret media have thus been widely used in applications where the pressure drop and/or energy conversation is critical, e.g., in heating, ventilating, and air conditioning (HVAC) systems, and in personal protective equipment (PPE), such as surgical masks and respirators. Composite media composed of multiple layers of electret media (in different specifications) have been found to offer better filtration performance than that of homogeneous electret media (Leung et al., 2009; Leung et al., 2018; Tang et al., 2018a; Chang et al., 2019; Sun and Leung, 2019; Tien et al., 2020). In our previous study (Wang et al., 2020), the initial collection efficiency of composite media made of multiple electret medium layers of the same specification were measured using size-fractionated particles in the Boltzmann stationary charge distribution. It was found that the measured filtration efficiency of the multi-layered composite electret media was lower than the calculated value, assuming each electret medium layer functions independently. The discrepancy observed was reduced as the particle size was increased. The study conducted by Sun and Leung (2019) also found that the figure of merit (FOM) of multi-layered electret media was less than that of base electret media. Moreover, the observed FOM difference was also reduced as the particle size was increased. However, if each electret medium layer functions independently, the FOM of composite media made of multi-layered electret media of the same specification should be the same as that of homogeneous media. The objective of this work is thus to find out the reasoning behind the previously reported discrepancy.

After examining all the possibilities, we hypothesized that the reported discrepancy between the measured and calculated collection efficiencies of multi-layered composite electret media was due to the electrical charge status of the test particles. Note that the effect of particle charge on the performance of electret media has already been widely investigated (Kanaoka et al., 1987; Fjeld and Owens, 1988; Otani et al., 1993; Chen and Huang, 1998; Romay et al., 1998; Yang et al., 2007; Chazelet et al., 2011; Sanchez et al., 2013; Tang et al., 2018b) and that this study is, by no 
Table 1. The specification of selected electret media in two kinds as the medium layers for tested composite media.

\begin{tabular}{lllll}
\hline Media & Thickness $(\mathrm{mm})$ & Fiber diameter $(\mu \mathrm{m})$ & Packing density & Initial pressure@10 $\mathrm{cm} \mathrm{s}^{-1}(\mathrm{~Pa})$ \\
\hline A & 0.55 & $15.6 \pm 1.04$ & 0.140 & 8.9 \\
B & 0.62 & $3.0 \pm 0.82$ & 0.100 & 48.2 \\
\hline
\end{tabular}

means, to repeat the previous works.

To validate our hypothesis, composite media composed of multiple electret medium layers with the same specification were tested. Two electret media, one used by $3 \mathrm{M}$ respirators and the other used by HVAC filter panels (rated at MERV 13), were selected as base layers. The specification of the selected base electret media is given in Table 1 for reference. Size-fractionated particles with single charges, no charges and the Boltzmann charge distribution were prepared as test particles. The measured collection efficiency of the double-layered media was then compared to that of the calculated value based on the measured collection efficiency of the base medium layers. The experimental setup and result are presented in the following sections.

\section{METHODS}

Fig. 1 shows the schematic diagram of the experimental setup to measure the size-fractionated filtration efficiency of the composite electret media and base electret medium layers. In addition to polydisperse $\mathrm{NaCl}$ particles produced by a custom-made Collison atomizer, polydisperse silver particles were prepared by the evaporation-condensation method (Scheibel and Porstendo, 1983). The size distributions of the generated $\mathrm{NaCl}$ and silver particles can be found in Fig. S1. Size-fractionated particles were then obtained by classifying the generated polydisperse particles with a differential mobility analyzer (DMA; Models 3081 and 3085; TSI Inc.). The sheath-toaerosol flow rate ratio was kept at 1:10 for the DMA operation. $\mathrm{NaCl}$ particles in the sizes of 50, $75,100,150,200,300,400$, and $500 \mathrm{~nm}$, and silver particles with the sizes of 10, 20, and $30 \mathrm{~nm}$ were selected as test particles. For each selected size, particles were prepared in three different charge statuses: singly charged, neutral, and Boltzmann-charge-distributed. Particles sized by the DMA were assumed to be singly charged because multiple-charged particles were minimized by selecting the particles from the right-hand side of the polydisperse particle size distribution modes. Particles with the Boltzmann stationary charge distribution were obtained by passing DMA-classified particles through a ${ }^{210}$ Po bipolar charger. Neutral particles were then prepared by passing particles with the Boltzmann charge distribution through an electrostatic precipitator (ESP) to remove the charged particles. The test filter media was placed in the filter holder and tested at the face velocity of $10 \mathrm{~cm} \mathrm{~s}^{-1}$, which is approximately the testing velocity for testing the particle collection efficiency of respirators based on the 42 CFR Part 84 regulation. An ultrafine condensation particle counter (UCPC; Model 3776; TSI Inc.) was used in the setup to measure the number concentrations of test particles upstream and downstream of the filter holder. A 3-way valve was used to switch the above upstream and downstream samplings.

Another ESP was also included in front of the UCPC when particles in the Boltzmann stationary charge distribution were chosen for the experimental runs. With the ESP turned on/off, the number concentration of both the neutral and total particles in the sample could be measured. The neutral fraction of sampled particles both upstream and downstream of the filter holder could then be calculated. Stainless-steel tubes were used in the setup to minimize the possible loss of charged particles in the transport lines.

\section{RESULTS AND DISCUSSION}

Assuming each base electret media layer functions independently, the size-fractionated collection efficiency of the double-layered electret media should be given as (Wang et al., 2008):

$E_{\text {double }}=1-P_{\text {double }}=1-P_{1} \times P_{2}$

where $P_{1}$ and $P_{2}$ are the penetration measured for each base layer, and $P_{\text {double }}$ and $E_{\text {double }}$ are the 


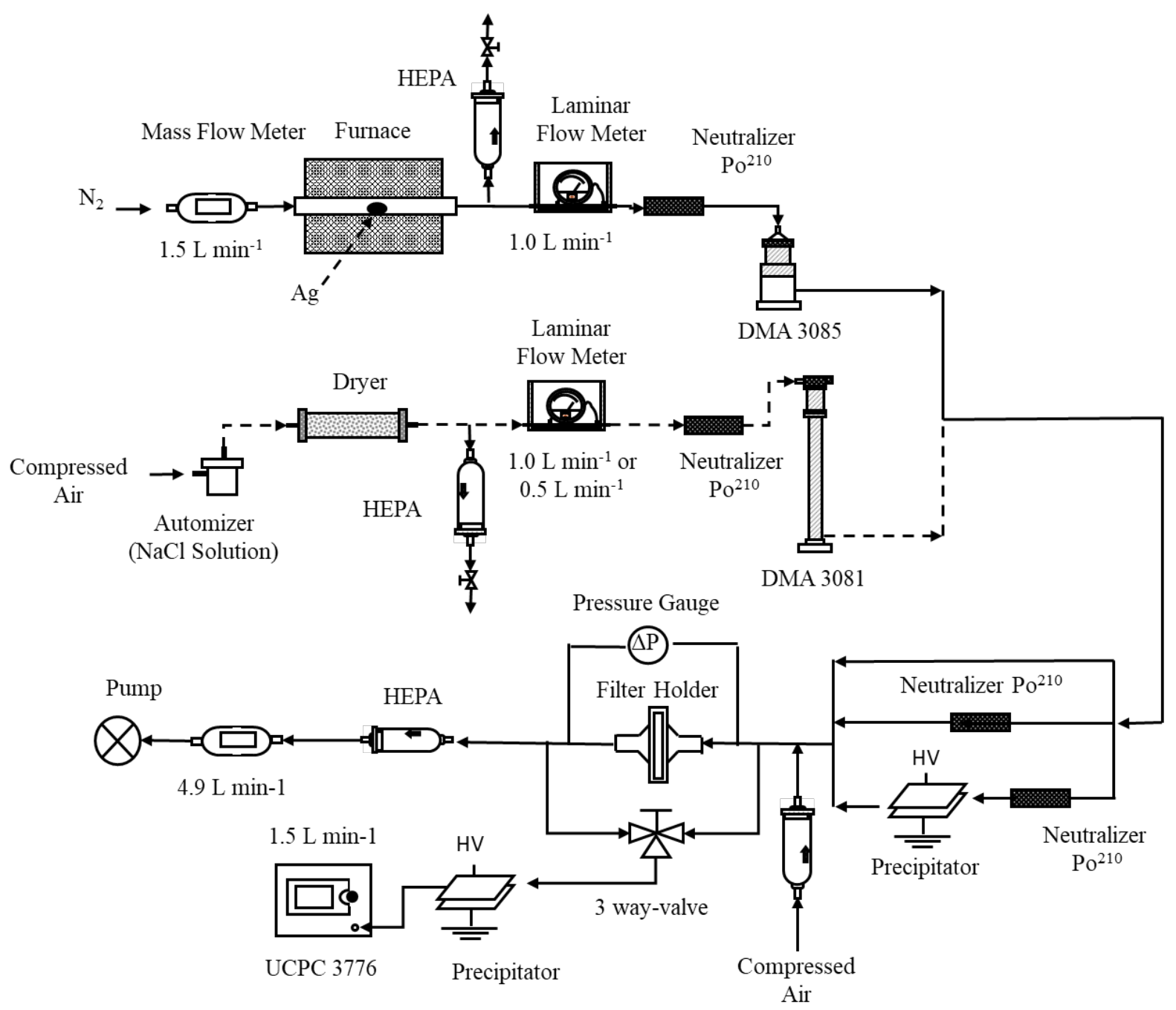

Fig. 1. The schematic diagram of the experimental setup for measuring the filtration efficiency of composite media composed of multiple-layered and base electret media.

penetration and collection efficiency of double-layered composite media, respectively. Below, we compare the measured data with that calculated by Eq. (1) using the measured penetration efficiency of base layers. Note that three media samples were tested in each experimental run and the average of measured efficiency data was shown in the following figures (i.e., Figs. 2-5). Error bars associated with each average data shown in figures represents the variation of the particle collection efficiency of tested media due to its nonuniformity.

Fig. 2 shows the comparison between the measured and calculated collection efficiencies of double-layered electret media when using test particles of both neutral (Fig. 2(a)) and singly charged (Fig. 2(b)) status. It is found that the agreement between the measured and calculated values was very good, indicating each base media layer indeed functioned independently in the double-layered composite media.

Fig. 3 shows the comparison between the measured and calculated collection efficiencies of the double-layered electret media when challenged by particles with the Boltzmann stationary charge distribution. Differences between the measured and calculated data were only observed in a certain size range of particles (as reported by Wang et al., 2020) for both double-layered electret media.

To further investigate the observation shown in Fig. 3, we measured the neutral fraction of test particles upstream and downstream of the filter holder by turning the ESP in front of the UCPC on and off. Fig. 4 shows the neutral faction of particles sampled upstream and downstream of the base electret media $A$ and $B$ at different particle sizes. For reference, the neutral fraction 


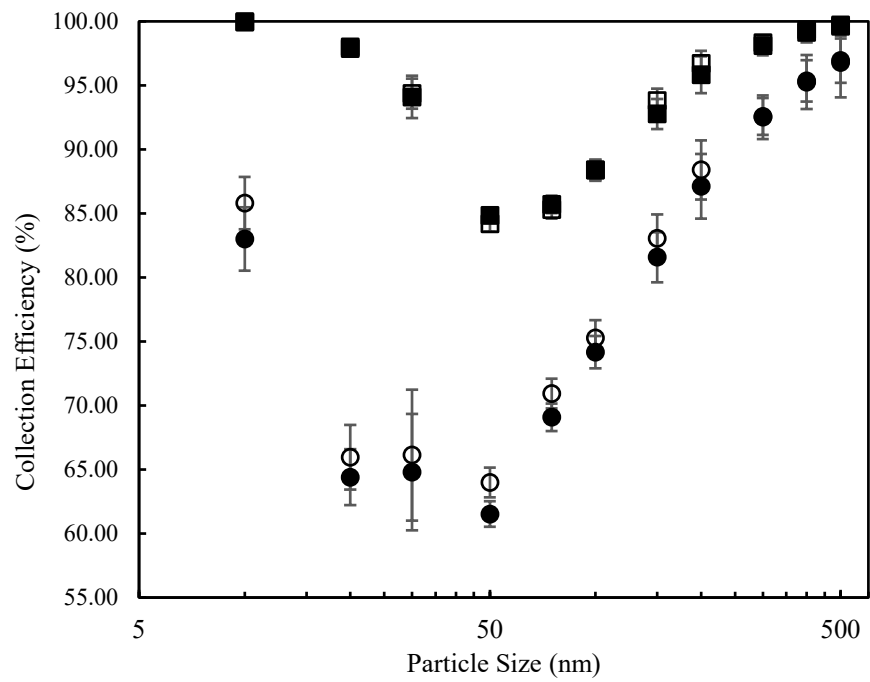

(a)

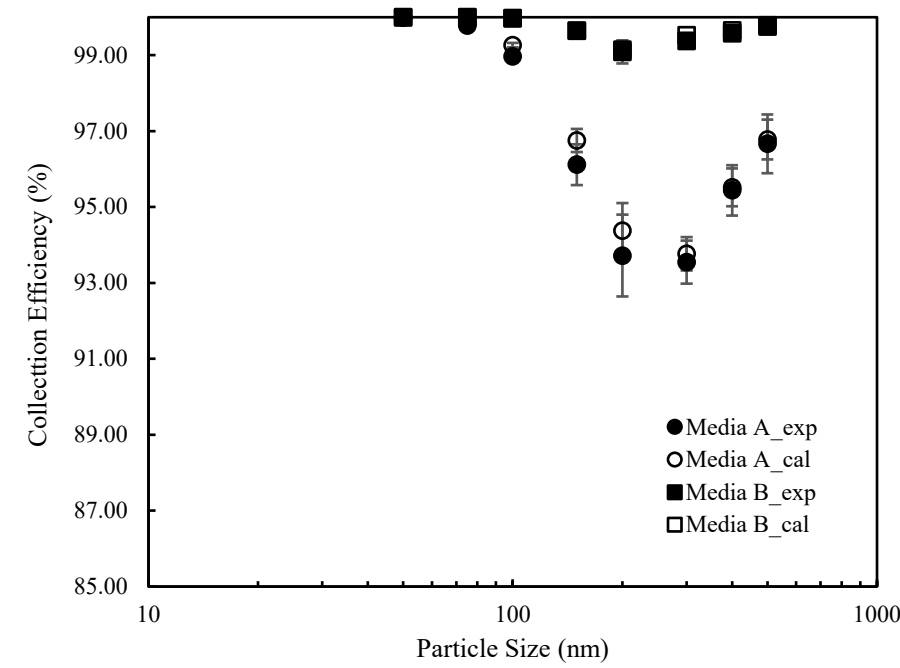

(b)

Fig. 2. The comparation between the measured and calculated particle collection efficiency of tested composite media when challenged by (a) neutral particles and (b) singly charged particles.

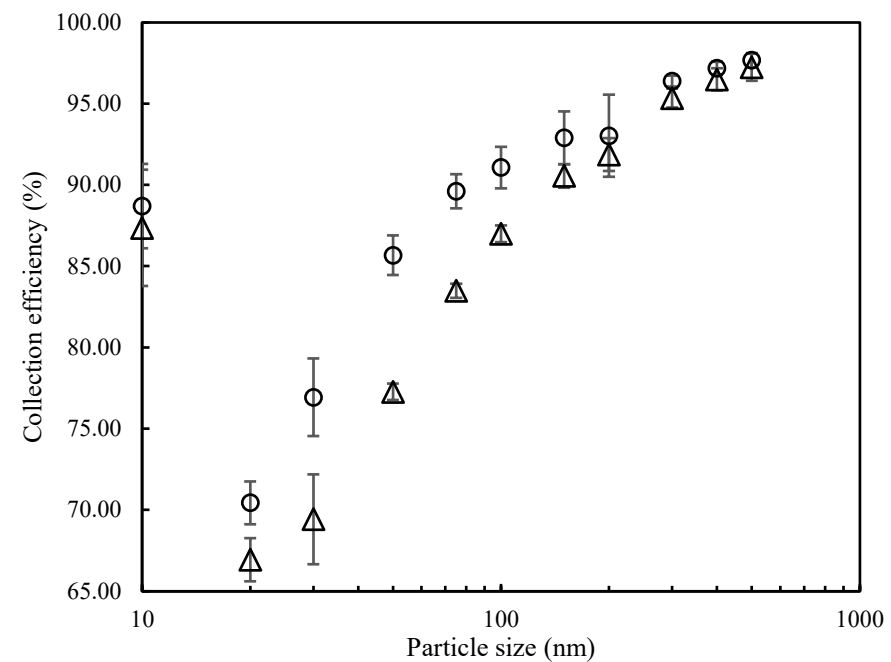

(a)

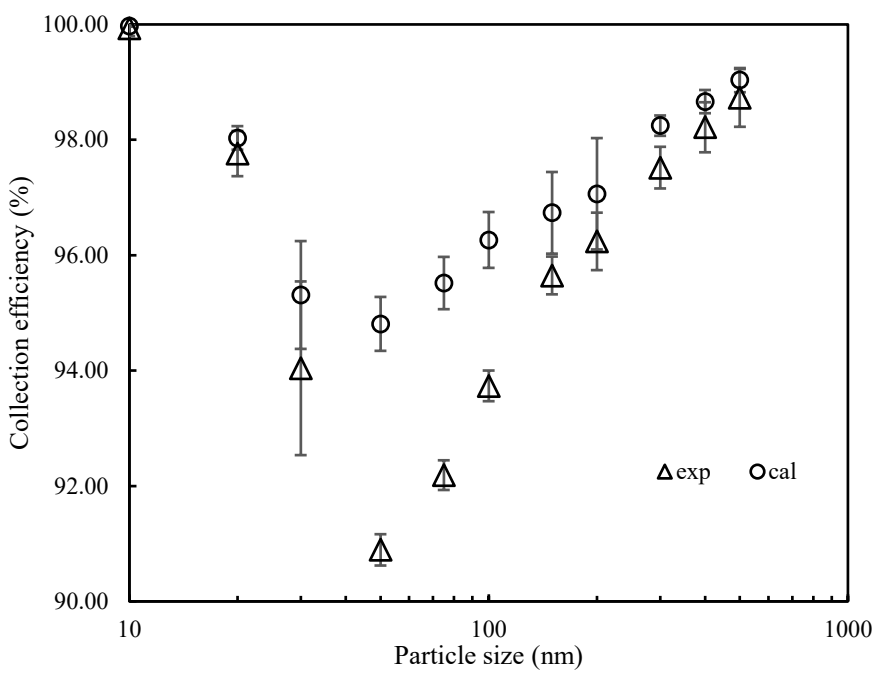

(b)

Fig. 3. The comparation between the measured and calculated particle collection efficiency of tested composite media when challenged by particles in Boltzmann stationary charge distribution for electret medium layers (a) A and (b) B.

of particles obtained from the theoretical Boltzmann charge distribution is also included in the figure. The charges of the upstream particles were indeed in the Boltzmann stationary charge distribution, but this was not the case for the downstream particles within a certain size range. For particles less than $30 \mathrm{~nm}$ in size, only neutral particles were present downstream of the filter media. As the particle size increased, the neutral fraction of downstream particles was reduced, but remained higher than the value given by the theoretical Boltzmann charge distribution, until the size of $\sim 500 \mathrm{~nm}$. This result is due to the collection of more charged particles than neutral ones in the electret media as test particles were passed. Notice that the neutral fraction difference between the upstream and downstream particles is negligible as the particle size is reduced to less than $10 \mathrm{~nm}$, because the charged fraction of particles in the Boltzmann charge distribution is negligible in that range (e.g., $0.7 \%$ for $10 \mathrm{~nm}$ particles). For particles of large sizes, their capture by the electrostatic mechanisms in electret media is greatly reduced due to reduced electrical mobility (shown in Fig. S2 in SI). The charge status of the downstream particles for large-sized particles was thus close to the Boltzmann charge distribution. 
Based on the findings shown in Fig. 4, the calculated collection efficiency of the double-layered electret media was corrected by considering the charge distribution of test particles downstream of the base layers. Specifically, the collection efficiencies of the first basic electret media layer for both neutral and charged particles could be calculated by separately measuring the number concentrations of both neutral and charged particles in the upstream and downstream of the media layer (via turning on/off the electrostatic precipitator installed in front of the CPC shown in the setup). Given the known charge distribution of particles in the upstream of the second basic media layer, the collection efficiencies of the second layer could be measured. The above efficiency data were then used to determine the corrected collection efficiency of double-layered electret media. Fig. 5 shows the comparison between the measured and corrected collection efficiency of double-layered electret media made of the base medium layers A and B. The corrected efficiency values are now in good agreement with the measured ones for particles with the Boltzmann stationary charge distribution. For reference, the measured particle filtration efficiency of the first electret medium layer and the calculated efficiency of the second electret

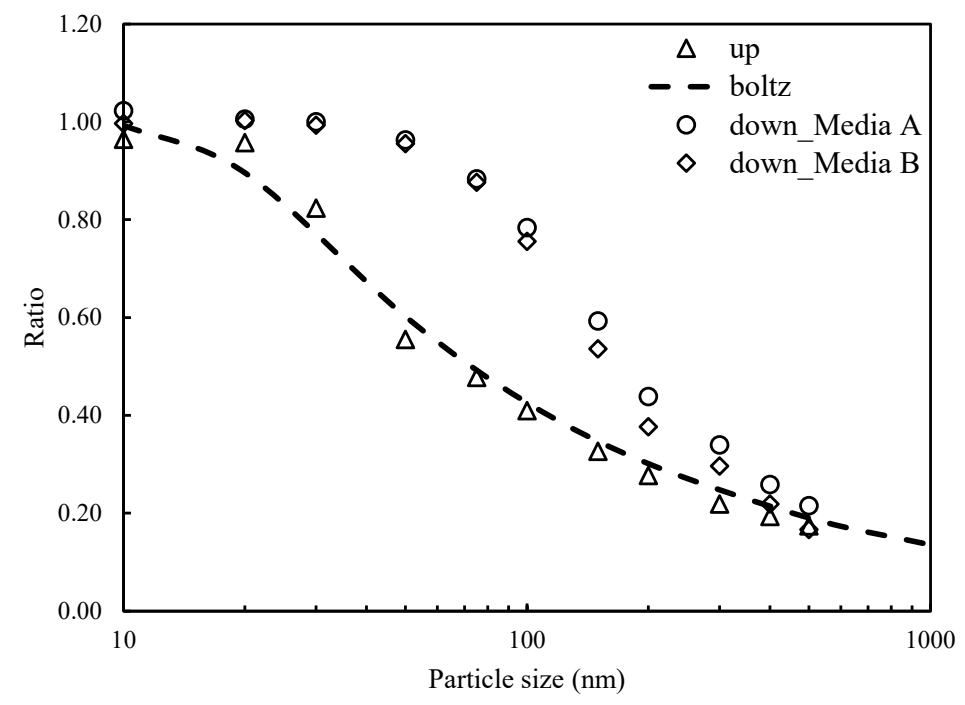

Fig. 4. The neutral fraction of test particles at the upstream and downstream of base electret medium layers.

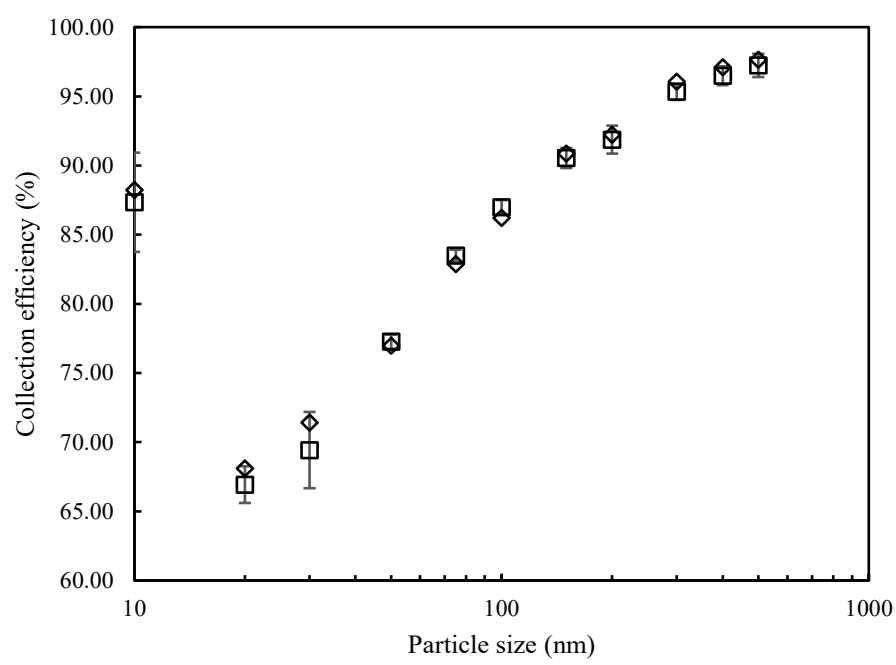

(a)

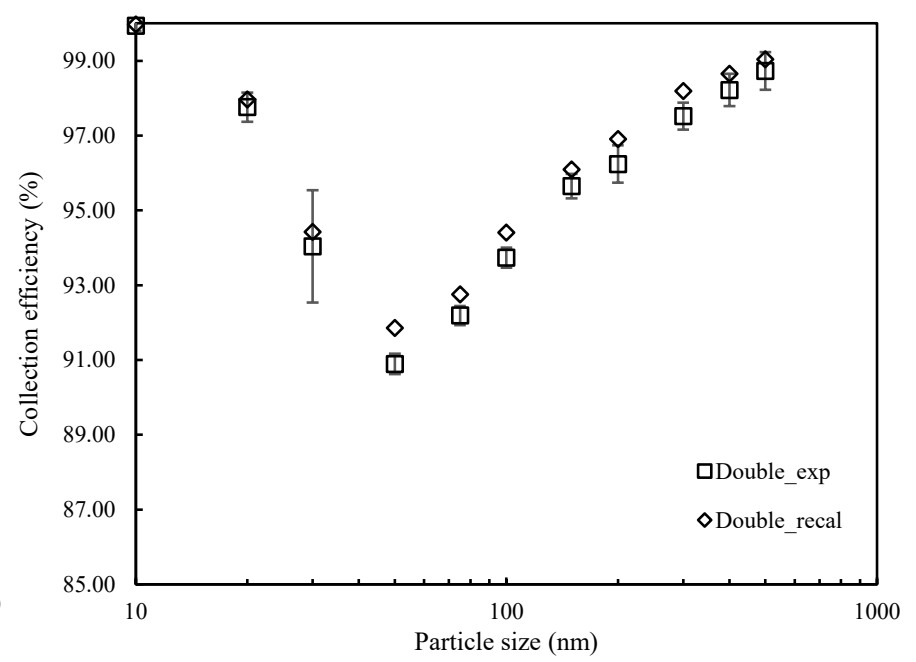

(b)

Fig. 5. The comparation between the measured and calculated particle collection efficiency of tested double-layered media in the case of using particles in the Boltzmann stationary charge distribution (after taking the charge distribution of test particles into the consideration) for base media (a) A and (b) B. 
medium layer (with the added consideration of particle charge distribution) for each composite electret media can be found in Fig. S3. These efficiency values were used in the calculation for the data presented in Fig. 5.

\section{FINAL REMARKS}

We resolved the discrepancy between the measured and the calculated filtration efficiencies of a composite formed of multiple electret medium layers, which has been reported in previous studies. We can accurately estimate the composite's filtration efficiency by assuming that each of its layers independently removes particles, but we must also account for the electrical charge distribution of the particles.

Owing to the difficulty in directly measuring the surface charge density of electret medium fibers, researchers typically obtain an estimate by comparing the calculated filtration efficiency (derived with an existing model) with observational data in the literature. Our findings suggest that such estimates should also factor in the charge distribution of the particles. For the sake of simplicity, the filtration data for either neutral or single-charged particles may be applied.

\section{SUPPLEMENTARY MATERIAL}

Supplementary data associated with this article can be found in the online version at https://doi.org/10.4209/aaqr.210005

\section{REFERENCES}

Chang, D.Q., Tien, C.Y., Peng, C.Y., Tang, M., Chen, S.C. (2019). Development of composite filters with high efficiency, low pressure drop, and high holding capacity $\mathrm{PM}_{2.5}$ filtration. Sep. Purif. Technol. 212, 699-708. https://doi.org/10.1016/j.seppur.2018.11.068

Chazelet, S., Bemer, D., Grippari, F. (2011). Effect of the test aerosol charge on the penetration through electret filter. Sep. Purif. Technol. 79, 352-356. https://doi.org/10.1016/j.seppur.201 1.03.021

Chen, C.C., Huang, S.H. (1998). The effects of particle charge on the performance of a filtering facepiece. Am. Ind. Hyg. Assoc. J. 59, 227-233. https://doi.org/10.3320/1.2765005

Fjeld, R.A., Owens, T.M. (1988). The effect of particle charge on penetration in an electret filter. ITIA 24, 725-731. https://doi.org/10.1109/28.6128

Kanaoka, C., Emi, H., Otani, Y., liyama, T. (1987). Effect of charging state of particles on electret filtration. Aerosol Sci. Technol. 7, 1-13. https://doi.org/10.1080/02786828708959142

Leung, W.W.F., Hau, C.W.Y., Choy, H.F. (2018). Microfiber-nanofiber composite filter for highefficiency and low pressure drop under nano-aerosol loading. Sep. Purif. Technol. 206, 26-38. https://doi.org/10.1016/j.seppur.2018.05.033

Leung, W.W.F., Hung, C.H., Yuen, P.T. (2009). Experimental investigation on continuous filtration of sub-micron aerosol by filter composed of dual-layers including a nanofiber layer. Aerosol Sci. Technol. 43, 1174-1183. https://doi.org/10.1080/02786820903261086

Otani, Y., Emi, H., Mori, J. (1993). Initial collection efficiency of electret filter and its durability for solid and liquid particles [translated]. Kona Powder Part. J. 11, 207-214. https://doi.org/10.14 356/kona.1993023

Romay, F.J., Liu, B.Y., Chae, S.J. (1998). Experimental study of electrostatic capture mechanisms in commercial electret filters. Aerosol Sci. Technol. 28, 224-234. https://doi.org/10.1080/0278 6829808965523

Sanchez, A.L., Hubbard, J.A., Dellinger, J.G., Servantes, B.L. (2013). Experimental study of electrostatic aerosol filtration at moderate filter face velocity. Aerosol Sci. Technol. 47, 606615. https://doi.org/10.1080/02786826.2013.778384

Scheibel, H., Porstendo, J. (1983). Generation of monodisperse Ag-and $\mathrm{NaCl}$-aerosols with particle diameters between 2 and $300 \mathrm{~nm}$. J. Aerosol Sci 14, 113-126. https://doi.org/10.1016/ 0021-8502(83)90035-6 
Sun, Q., Leung, W.W.F. (2019). Charged PVDF multi-layer filters with enhanced filtration performance for filtering nano-aerosols. Sep. Purif. Technol. 212, 854-876. https://doi.org/10. 1016/j.seppur.2018.11.063

Tang, M., Chen, S.C., Chang, D.Q., Xie, X., Sun, J., Pui, D.Y. (2018a). Filtration efficiency and loading characteristics of $\mathrm{PM}_{2.5}$ through composite filter media consisting of commercial HVAC electret media and nanofiber layer. Sep. Purif. Technol. 198, 137-145. https://doi.org/10.1016/j.sepp ur.2017.03.040

Tang, M., Thompson, D., Chang, D.-Q., Chen, S.C., Pui, D.Y. (2018b). Filtration efficiency and loading characteristics of $\mathrm{PM}_{2.5}$ through commercial electret filter media. Sep. Purif. Technol. 195, 101-109. https://doi.org/10.1016/j.seppur.2017.11.067

Tien, C.Y., Chen, J.P., Li, S., Li, Z., Zheng, Y.M., Peng, A.S., Zhou, F., Tsai, C.J., Chen, S.C. (2020). Experimental and theoretical analysis of loading characteristics of different electret media with various properties toward the design of ideal depth filtration for nanoparticles and fine particles. Sep. Purif. Technol. 233, 116002. https://doi.org/10.1016/j.seppur.2019.116002

Wang, J., Kim, S.C., Pui, D.Y. (2008). Investigation of the figure of merit for filters with a single nanofiber layer on a substrate. J. Aerosol Sci 39, 323-334. https://doi.org/10.1016/j.jaerosci.2 007.12.003

Wang, P., Liu, Z., Chen, D.R. (2020). Performance of composite filters assembled from multiple layers of basic filtration media. Aerosol Air Qual. Res. 20, 2299-2308. https://doi.org/10.4209/ aaqr.2020.07.0368

Yang, S., Lee, W.M., Huang, H.L., Huang, Y.C., Luo, C.H., Wu, C.C., Yu, K.P. (2007). Aerosol penetration properties of an electret filter with submicron aerosols with various operating factors. J. Environ. Sci. Health A 42, 51-57. https://doi.org/10.1080/10934520601015651 